\title{
Simultaneous color contrast from McCollough effects is spatially contingent
}

\author{
W. R. WEBSTER \\ Monash University, Clayton, Victoria, Australia \\ B. CRASSINI \\ University of Queensland, St. Lucia, Queensland, Australia \\ and \\ K. WILLENBERG \\ Monash University, Clayton, Victoria, Australia
}

\begin{abstract}
In two experiments, orientation-contingent color aftereffects (CAEs) were induced by presenting in alternation red/black vertical and green/black horizontal gratings. The CAEs induced secondorder simultaneous color contrast in a $45^{\circ}$ patterned test field but not in a patternless field. These CAE-induced second-order color effects were measured using both color cancellation (Experiment 1) and color matching (Experiment 2) techniques. The spatial selectivity of the second-order effects and their manifestation outside the orientation range typically used in studies of orientationcontingent color effects are discussed in terms of neural explanations of color misperceptions.
\end{abstract}

An achromatic unpatterned field embedded in a colored surround is seen as colored, the apparent hue being approximately complementary to the color of the surround. This compelling phenomenon is called simultaneous color contrast, and has a long history in the perceptual literature (Graham \& Brown, 1965). An equally compelling but more recently discovered misperception of color occurs after repeated presentation of stimuli of particular hues and orientations, for example, vertical contours in red light and horizontal contours in green light. If two such stimuli are observed in alternation for a few minutes, and then achromatic test stimuli of the same orientation as the former are viewed, then they appear colored. In the above example, achromatic vertical contours would appear green, and achromatic horizontal contours would appear red. These colored aftereffects (CAEs), first reported by McCollough (1965), are contingent on the retinal orientation of the achromatic test stimuli. The CAEs reverse when the head is tilted through $90^{\circ}$ so that a grating vertical with respect to gravity is now horizontal with respect to the retina.

Stromeyer $(1978,1984)$ reported that simultaneous color contrast can be produced by an achromatic stimulus that appears colored. For example, after generating CAEs by inspecting vertical and horizontal colored stimuli, Stromeyer (1984) found that McCollough effects induced simultaneous color contrast in a diagonal achromatic test grating placed between either two vertical or two horizontal achromatic gratings. The simultaneous color contrast so produced varied as a function of both

Address correspondence to W. R. Webster, Neuropsychology Laboratory, Department of Psychology, Monash University, Clayton, Victoria 3168, Australia. the spatial frequency and the orientation of the embedded center grating. Thus a center grating tilted $22.5^{\circ}$ from the surrounding vertical gratings had no color, but a grating tilted farther from vertical appeared to have a color complementary to the CAEs in the surrounding gratings. Stromeyer's (1984) results were based on data from only himself and one other subject. Furthermore, he used a rating method based on a matching color task, rather than a more quantitative measure of the colored effects, such as color cancellation. In the two experiments reported here we confirm and extend Stromeyer's (1984) observation, using color cancellation techniques. Our results demonstrate that simultaneous color contrast and CAEs are related, and that color contrast induced by CAEs is reported only when the test stimulus is patterned.

CAE-induced simultaneous color contrast may be termed a second-order effect (see Anstis, Rogers, \& Henry, 1978), because the distortion in color appearance of a test stimulus is dependent on the occurrence of another distortion in color appearance (in this case, a CAE). A similar second-order color effect was first described by Purkinje in the early 1880s and has been rediscovered a number of times since. Anstis et al. (1978) reported that inspection of a colored adaptation field with a gray center region resulted in three misperceptions of color: The gray center region appeared colored (simultaneous color contrast); a gray test field observed following inspection of the adaptation field appeared colored (negative afterimage); and the center of this negative afterimage, corresponding to the location of the gray patch in the original display, also appeared colored, but in the hue opposite that of the negative afterimage. Anstis et al. (1978) found that this second-order effect was a function of both simul- 
taneous color contrast induced by the first-order afterimage of the surround and an afterimage induced by the first-order simultaneous color contrast in the original display. The occurrence of second-order color misperceptions was taken by Anstis et al. (1978) to suggest "the existence of recurrent or feedback loops in the visual system, or else that adaptation processes occur at different sites both before and after lateral inhibitory processes" (p. 911).

Similar neural processes form the basis of physiological explanations of CAEs, except that rather than occurring within a "color system" they are taken to involve mechanisms sensitive to narrow regions of both wavelength and spatial dimensions (Harris, 1980; Over, 1977; Stromeyer, 1978). This assumption is necessary to account for the spatial selectivity of CAEs. For example, color-orientation stimuli used to induce orientationcontingent CAEs must differ by more than $22^{\circ}$, and the most saturated CAEs are reported when test stimuli are identical in orientation to the adaptation stimuli. As test stimuli are shifted in orientation away from the adaptation values, CAEs become weaker. For example, a $45^{\circ}$ test stimulus used after adaptation to vertical and horizontal stimuli is reported as achromatic (Stromeyer, 1978). The orientation selectivity demonstrated during adaptation and test phases of CAEs can be understood in terms of the activity of mechanisms sensitive to limited regions of the orientation domain, that is, mechanisms that have a preferred orientation and respond at a systematically decreasing rate over a $\pm 20^{\circ}$ tuning range. With such orientation tuning, the selective adaptation necessary to explain CAEs generated by using the typical dual adaptation procedure (i.e., one orientation in red, another in green) can occur only if adaptation stimuli differ by more than $20^{\circ}$. Similarly, test stimuli differing by more than $20^{\circ}$ from the adaptation stimuli will be processed by mechanisms uninfluenced by the adaptation stimuli, and will therefore not elicit CAEs.

In the present experiments, second-order color effects induced by CAEs were demonstrated by use of a test stimulus consisting of an oblique grating. Vertical and horizontal contours were used to generate the CAEs; therefore, the second-order effects were manifested in the test stimuli outside the orientation tuning range normally necessary to elicit CAEs. This fact, together with the spatial dependence of second-order effects induced by CAEs, has implications for neural explanations of misperceptions of color. These implications are discussed below.

\section{EXPERIMENT 1}

\section{Method}

Subjects. Seven graduate students acted as volunteer subjects. All had normal visual acuity and normal color vision.

Apparatus and Stimuli. All stimuli were presented on a Barco color monitor (Model GD 33), using a real-time image processing system (Image Technology Inc., Model AP-512) interfaced to a computer (DEC LSI-11/73). Instructions to subjects and monitoring of responses (a series of button presses; see below) were also under computer control.

Adaptation stimuli consisted of square-wave gratings (spatial frequency $2 \mathrm{cpd}$ ) presented vertically in red light (RV; dominant wavelength $620 \mathrm{~nm}$ ) or horizontally in green light (GH; dominant wavelength $515 \mathrm{~nm}$, as measured by a Minolta Chromameter, Model CL100). The gratings subtended $11.9^{\circ} \times 10.2^{\circ}$ visual angle and were presented at an average luminance of $17.3 \mathrm{~cd} / \mathrm{m}^{2}(\mathrm{RV})$ and $20.6 \mathrm{~cd} / \mathrm{m}^{2}(\mathrm{GH})$, as measured by a Minolta Auto-spot II light meter. The display used in the test phases consisted of two separate components. The surround subtended $14.6^{\circ} \times 10.2^{\circ}$ and was either a vertical or a horizontal grating $(2 \mathrm{cpd}$; average luminance $22.6 \mathrm{~cd} / \mathrm{m}^{2}$ ). Located centrally in the surround was a rectangular region, subtending $7.4^{\circ} \times 5.4^{\circ}$, which could be either an unpatterned field (average luminance $26.6 \mathrm{~cd} / \mathrm{m}^{2}$ ) or a $45^{\circ}$ oblique grating pattern of the same spatial frequency as the surround and with an average luminance of $23.3 \mathrm{~cd} / \mathrm{m}^{2}$.

The subjects could alter the color appearance of either the surround or center region of the test display by pressing one of three colored buttons (red, green, white) that controlled, through the computer, the combined output of the red (R), green (G), and blue (B) guns of the monitor. The test stimuli could be altered from a starting value at which the display was colorless (i.e., the R, G, and B guns' output was such that it produced white light), or red or green (i.e., the output of the $R$ or $G$ gun exceeded that of the others). The test stimuli could also be altered from a starting value at which the display was actually achromatic but appeared colored (e.g., after RV and GH adaptation). In the cases where the stimuli were or appeared colored, the subjects could cancel the color appearance (i.e., make the display appear achromatic). For example, if the test stimulus looked red, the subject could press the red button to decrease the saturation of the color with each button press. This continued until the stimulus appeared colorless, at which point the subject pressed the white button. Continued pressing of the white button increased the green appearance of the stimulus, and when the subject decided that the display in fact looked green, he/she pressed the green button. The outputs of the R, G, and B guns of the monitor were yoked by software so that the relationship between gun output and button pressing was linear. The change in color appearance associated with each button press was below threshold as judged by the three authors. This ensured that the subjects could not use a response strategy based on counting of button presses. The principal advantage of this system is that it allows the independent cancellation of each grating, which is not possible with other color cancellation methods (Riggs, White, \& Eimas, 1974).

Procedure. The subjects sat $1 \mathrm{~m}$ from the Barco monitor and viewed the screen binocularly with heads upright throughout the experiment. Instructions outlining adaptation and test procedures were presented on the screen and an experimenter was available to answer questions. At the beginning of each session the subjects were given practice at setting colored test stimuli to white. After this practice, pretest measures were obtained in which achromatic test stimuli were presented and the subjects were required to set these to white. Since most subjects saw the pretest stimuli as white, their initial responses (which had to be either red or green) were guesses. Following this initial response, the subjects pressed the white button if the stimulus appeared achromatic and continued to press the white button until the stimulus first appeared colored (i.e., in the hue opposite that of the initial button press). For those subjects who saw color during the pretest, the initial response was to press the appropriate button (e.g., green if the stimulus looked green) and continue pressing until the stimulus appeared white. From this point the procedure was the same as described above. The test procedure provided three types of data for each subject: (1) the initial color appearance of the test stimulus, (2) the amount of color needed to cancel the initial color appearance, and (3) the amount of color 
needed to make the "cancelled" test stimulus appear to be colored with the color opposite to the initial color. These three measures were obtained for the eight test stimuli formed by the factorial combination of the two test locations (surround, center), the two surround orientations (vertical, horizontal), and the two types of center (unpatterned, oblique pattern). In addition, pre- and posttest measures were obtained with the two centers (unpatterned, oblique pattern) presented without the vertical and horizontal surrounds.

Following the pretest measurement, the subjects were exposed to the RV and GH adaptation stimuli in alternation (each presented for $7 \mathrm{sec}$ ) for a period of $5 \mathrm{~min}$. There was a 1 -min interval to allow dissipation of any afterimages before posttest measures were taken. The order of presentation of test stimuli was randomized across subjects for both pre- and posttest measures. After posttest measures were obtained, the subjects were asked to tilt their heads from side to side through $90^{\circ}$ and to report whether they saw any color changes in either surround or center.

\section{Results and Discussion}

Analyses were performed on only one of the three measures obtained during the test phases of the experimentthe amount of red or green added to the test stimulus to cancel its initial color appearance. An arbitrary signing system was adopted in treating the data. When the subjects saw a test stimulus as green and added red to produce an achromatic display, responses were scored as positive; when the test stimulus appeared red and green was added, responses were scored as negative. For each subject, pretest scores were subtracted from posttest scores to provide net color scores for each test stimulus. Separate three-way within-subjects analyses of variance (ANOVAs) were carried out on the surround data and on the center data.

The results show a significant difference between the color cancellation scores for vertical and horizontal surrounds $[F(1,6)=64.4, p<.01]$, whether the center was an oblique grating or a contourless white field. That is, a clear McCollough effect had been induced in each surround: vertical surrounds appeared green and horizontal surrounds appeared red, and each required addition of the opposite color to cancel to white (Figures 1A and 1B). There was no difference between surrounds as a function of center type [collapsed over orientation, $F(1,6)=0.0$, $p>.05]$. There was no significant interaction, and the plots of surround cancellation values were virtually identical for each center type (Figures 1A and 1B).

Although the color appearance of the surrounds was consistent, and was as expected on the basis of the colororientation combinations used during adaptation, the color responses to the center test stimuli were inconsistent. The color appearance of the test center when it contained oblique contours was dependent on the surround. When the surround was vertical (and appeared green) the contoured center appeared red and required the addition of green to cancel its color appearance (Figure 1A). The opposite was the case when the surround was horizontal. When the center was white and contourless, the orientation of the surround had no influence on its color appear- ance, and it was reported by subjects as being colorless (Figure 1B).

The ANOVA confirmed this interpretation. There was a significant effect of surround orientation on the color of the centers $[F(1,6)=72.41, p<.01]$. There was no significant difference beween center types when collapsed over orientation $[F(1,6)=2.41, p>.05]$, a result largely due to arithmetical cancellation of scores arbitrarily classified as either negative or positive. However, there was a significant interaction between surround orientation and centers $[F(1,6)=28.00, p<.01]$, indicating that the effect of surround orientation was different for the two types of centers. The nature of this difference can be seen clearly if the plots for the oblique field centers in Figure 1A are compared with plots for the white field centers in Figure 1B. This interpretation is supported by post hoc $t$ tests, which showed that the mean scores of the patternless centers were not significantly different from zero. A pre- and posttest measure was obtained for each center when it was presented without any surround on a black background. Individual $t$ tests between these means revealed no significant differences, indicating that no color changes were induced in either type of center when they were not set in patterned surrounds able to elicit CAEs.

When subjects were asked to tilt their heads from side to side through $90^{\circ}$, they reported that the color of each surround reversed from red to green or vice versa. However, only the color of the patterned centers reversed; there was no change in the color appearance of the white centers.

\section{EXPERIMENT 2}

In Experiment 1, a color cancellation technique was used to measure the color appearance of the center and surround test stimuli. In Experiment 2, color cancellation was also used to obtain measures of the CAEs induced in the surrounds by the exposure to RV and GH. However, a color matching procedure was used to compare the color appearance of an embedded center with that of a center without surrounds. The purpose of Experiment 2 was to confirm the spatial selectivity of the second-order effects induced by CAEs shown in Experiment 1, and to demonstrate that these effects and their spatial selectivity were not dependent on a particular method of estimating color appearance, namely color cancellation.

\section{Method}

Subjects. The same 7 graduate students with normal color vision acted as subjects.

Apparatus, Stimuli, and Procedure. The apparatus was the same as that in the first experiment. However, the test stimuli and procedures were modified slightly. Rather than occupying the full screen, as in Experiment 1, the test stimuli in Experiment 2 were presented on half the screen. The surround was on the right half of the screen (spatial frequency $2 \mathrm{cpd}$, average luminance $26.6 \mathrm{~cd} / \mathrm{m}^{2}$ ), and it subtended $7.1^{\circ} \times 7.1^{\circ}$. Within the surround was a centrally lo- 


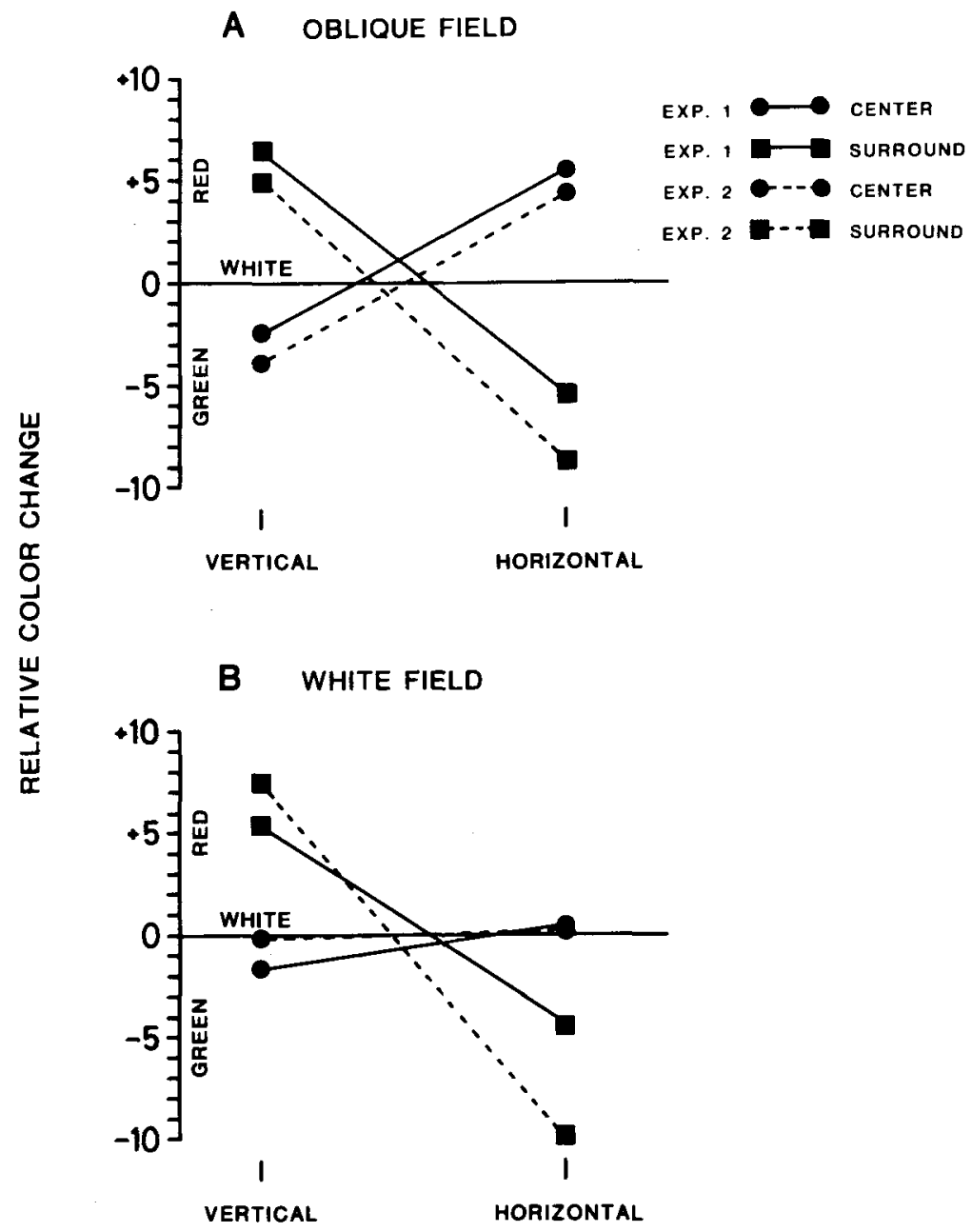

\section{SURROUND ORIENTATION}

Figure 1. Plots of means of color cancellation changes in Experiments 1 and 2 required to make an achromatic test stimulus appear white after adaptation to red vertical and green horizontal gratings. (A) Changes required to cancel surrounds and patterned oblique centers, both plotted as a function of surround orientation. Vertical surrounds required more red (positive score) and horizontal surrounds required more green (negative score). Patterned oblique centers required cancellation changes opposite to the particular surround in which they were embedded. (B) Changes for surrounds containing white patternless centers show results similar to those shown in A, but white patternless centers did not require any significant color change as a function of surrounding orientation.

cated rectangular test region that subtended $3.7^{\circ} \times 2.6^{\circ}$. The left half of the monitor screen was black except for a centrally located region of the same dimensions as the test center, and with the same spatial properties as the test center (i.e., it was an oblique grating pattern or a patternless region). This region was the color comparison stimulus.

The subject's task was either to cancel the color appearance of the surround by pressing the appropriate button (as in Experiment 1) or to match the color appearance of the comparison center by adjusting the color appearance of the embedded center. Because the comparison stimulus always appeared white, the response to the embedded center was in effect cancellation of any perceived color to white.
The same general procedures as those of Experiment 1 were used to obtain pretest and posttest measures. The pre- and posttest measures were again separated by $5 \mathrm{~min}$ of adaptation to RV and GH gratings of the same dimensions as in Experiment 1, but with exposures of $10 \mathrm{sec}$. An interval of $1 \mathrm{~min}$ was again allowed for any afterimages to dissipate. At the end of testing, the subjects were asked to tilt their heads through $90^{\circ}$ and to report any changes in color of either surround or center.

\section{Results and Discussion}

The responses to the colors of the surrounds and the centers were treated as in Experiment 1; that is, a posi- 
tive score indicated that the test stimulus appeared green and a negative score indicated red. Separate ANOVAs were again performed on surround and center scores.

The results of Experiment 2 were similar to those of Experiment 1. In the ANOVA on the surround data, a significant difference was obtained between the color cancellation scores for vertical and horizontal surrounds, thus indicating a strong McCollough effect $[F(1,6)=55.89$, $p<.01]$. The difference between surrounds as a function of center type was not significant $[F(1,6)=0.77$, $p>.05]$. The interaction between surround and center also failed to reach significance $[F(1,6)=4.48, p>.05]$. There was again an influence of surround color on the color of oblique centers when the subjects' task was color matching (see Figures 1A and 1B). The ANOVA performed on the center data showed that the effect of surround orientation on color responses to the centers was significant $[F(1,6)=52.02, p<.01]$, but that the difference between center types was not $[F(1,6)=0.07$, $p>.05]$. However, there was a significant interaction between surround orientation and centers $[F(1,6)=$ $52.02, p<.01]$, indicating that the effect of the surrounds differed for the two types of centers and was due to a change in the color appearance of the oblique patterned centers. This interpretation was supported by the fact that the mean scores of the contourless centers did not differ significantly (according to $t$ tests) from zero (see Figure 1B). There was no significant difference between pre- and posttest scores for the centers presented alone. Reversals in the colors of the surrounds and the oblique centers occurred when subjects rotated their heads through $90^{\circ}$.

These results confirm those of Experiment 1 in that the occurrence of second-order simultaneous color contrast effects was contingent on spatial properties of the stimulus. Only centers containing oblique contours were influenced by CAEs. As was the case in Experiment 1, the color induced in these centers was genuinely a secondorder effect dependent on the CAE in the surround, inasmuch as no color was observed in any center presented alone. The failure of patternless centers to elicit secondorder simultaneous color contrast did not occur when these centers were embedded in actually colored surrounds. When presented with such displays, all subjects reported first-order simultaneous color contrast in both types of center.

\section{GENERAL DISCUSSION}

The present results provide quantitative support for Stromeyer's (1984) observation that CAEs can induce simultaneous color contrast in a grating pattern flanked by two other grating patterns when the latter are colored due to CAEs. They also confirm Stromeyer's observation that simultaneous contrast is also a function of the orientation of the stimulus. A characteristic of CAEs is their spatial selectivity (see Over, 1977; Stromeyer, 1978), and any explanation of CAEs must take this spa- tial selectivity into account. The most frequently cited neural explanations of CAEs have assumed the existence of "double-duty" neurons (Over, 1977) that are sensitive to narrow regions of both the wavelength and spatial domains. Simultaneous color contrast, on the other hand, is considered to be an "areal" rather than a pattern effect. That is, it is manifested over an area, due to the influence of an adjacent region. The results of the present experiments indicate that a distinction should be drawn between first-order and second-order simultaneous color contrast effects: First-order effects may be considered in areal rather than pattern terms, whereas second-order color contrast effects are contingent on patterning of the test region.

Richards (1977) pointed out that first-order simultaneous color contrast effects would most likely be due to double-opponent process cells, such as the cells with concentric receptive fields found by Michael (1978a) in area 17 of the monkey. However, the present study suggests that the second-order simultaneous color contrast effects are mediated by cells that are both double-opponent process and orientation sensitive. The simple cells reported by Michael (1978b) possess these features. Indeed, Michael (1978b) suggested that these cells could well be the basis of the McCollough effects. For example, Michael (1978b) found that simple cells with a redon green-off central strip will, after exposure to a red and black grating, give " off" responses to a white bar of the appropriate orientation presented in the central strip of the receptive field. That is, after exposure to a red grating, these cells respond to achromatic gratings as if they were green. The activity of these cells parallels the experience of an observer reporting CAEs.

Although the activity of orientation-tuned doubleopponent process cells may provide the neural basis for CAEs, some modification to this explanation is required to account for second-order effects induced by CAEs. The data represented in Figure 1 show that such second-order effects occur in test stimuli with grating orientations outside the tuning range of those color-orientation mechanisms assumed to be processing (and therefore influenced by) the adaptation stimuli. Such test stimuli are ineffective in producing CAEs (Stromeyer, 1978). This failure of oblique patterns to elicit CAEs following verticalhorizontal CAE induction was confirmed in the present experiments. Presented alone, oblique centers appeared achromatic; presented with surrounds that appeared colored, oblique centers also appeared colored (see Figure 1). These results raise the question of how cells sensitive to either vertical or horizontal patterns can influence cells outside their tuning ranges. A possible answer is provided by some recent work on the cat visual cortex, which has shown that, for example, stimulation with a vertical grating can inhibit cells sensitive to a horizontal one (Morrone, Burr, \& Maffei, 1982). In fact, when such a cell is tuned for this inhibition, it is found that the inhibition begins to occur just outside the excitatory tuning range. This outcome indicates that cells with 
a preferred horizontal or vertical orientation may influence cells sensitive to oblique orientations. It is of importance, therefore, to determine whether similar interactions can be found for color-opponent simple cells in the monkey cortex, cells of the type reported by Michael (1978b). Such cells could be the neurophysiological basis of the second-order simultaneous contrast effects reported here.

Our data indicate that two processes are involved in simultaneous color contrast. One produces first-order effects when real colors in the surround produce simultaneous color contrast in both types of test center. The other produces the second-order effect when CAEs induce simultaneous color contrast. We have described first-order simultaneous color contrast as an areal or nonpattern phenomenon. The data in Figure 1 indicate that secondorder simultaneous color contrast is a pattern phenomenon. The implication of this distinction is that first-order effects may be mediated by a "color-only" system, whereas second-order effects may involve a "colorpattern' system.

It is usually argued that the McCollough effect results from an adaptation of cells sensitive to both color and orientation and that these cells are located in the cortex. However, Murch and Hirsch (1972) showed that the effect could be produced by another second-order misperception of color. They induced a CAE by superimposing afterimages on black-and-white vertical and horizontal gratings. Because afterimages are usually thought to be a retinal process (Craik, 1940), this means that retinabased effects must be combined in the cortex with spatial information. Murch and Hirsch's (1972) data suggest that the McCollough effect is due to an adaptation of a color system that is separate from an orientation-sensitive system. This suggestion is also supported by the observations of Ambler and Foreit (1978), who found that a patternless colored stimulus could be substituted for one grating to induce a weaker than normal McCollough effect. The cells proposed by Michael (1978b) as the basis of McCollough effects do not fire well to a uniformly colored field. For example, double-opponent simple cells do not fire at all to a large red or green band that covers the whole receptive field. This would suggest that the influence of both afterimages and patternless colored inducing fields must be taking place at a lower level of the system than the level of the cortical cells described by Michael (1978b). Thorell, De Valois, and Albrecht (1984) proposed that simple cells are "double opponent" with respect to both luminance and color; that is, simple cells are selective for both color and spatial frequency, and their receptive fields contain antagonistic regions for both types of stimulus. It is possible that both the second-order afterimage colors and real color fields can unite with spatial frequency information at lower levels, and that their combined output then influences these cortical cells to produce the McCollough effect.

Despite the results of Murch and Hirsch (1972), it is clear from the work of Harris and Gibson (1981) and Han- sel and Mahmud (1978) that negative afterimages are not the basis of CAEs. Hansel and Mahmud (1978) showed that opposite misperceptions of color (one an afterimage, the other an orientation-specific CAE) could be induced in the same retinal area. This indicates that the locus of the afterimage is different from the locus of the McCollough effect. Given current ideas concerning the parallel and serial processing of spatial and wavelength information (see, e.g., Desimone, Schein, Moran, \& Ungerleider, 1985), Hansel and Mahmud's (1978) claim cannot be dismissed.

In summary, an achromatic grating appears colored as a consequence of a color-spatial adaptation that can induce a second-order misperception of color in a patterned field, but not in an unpatterned field. This suggests the occurrence of inhibitory interactions in color-orientation mechanisms outside the commonly accepted orientation tuning ranges.

\section{REFERENCES}

AMBler, B. A., \& Foreit, K. G. (1978). Induction of the McCollough effect II: Two different mechanisms. Perception \& Psychophysics, 24, 466-470.

Anstis, S., Rogers, B., \& Henry, J. (1978). Interactions between simultaneous contrast and coloured afterimages. Vision Research, 18, 899-911.

Craik, K. J. W. (1940). Origin of visual afterimages. Nature, 145, 512-513.

Desimone, R., Schein, S. J., Moran, J., \& Ungerleider, L. G. (1985). Contour, color and shape analysis beyond the striate cortex. Vision Research, 25, 441-452.

Graham, C.H., \& Brown, J. L. (1965). Color contrast and color appearances: Brightness constancy and color constancy. In C. H. Graham (Ed.), Vision and visual perception. New York: Wiley.

Hansel, C. E. M., \& Mahmud, S. D. (1978). Comparable retention times for the negative color afterimage and the McCollough effect. Vision Research, 18, 1601-1605.

HARRIS, C. S. (1980). Insight or out of sight? Two examples of perceptual plasticity in the human adult. In C. S. Harris (Ed.), Visual coding and adaptability. Hillsdale, NJ: Erlbaum.

HARRIS, C. S., \& Gibson, A. R. (1981). Is orientation-specific color in human vision due to edge detectors, after-images or "dipoles"? Science, 162, 1506-1507.

MCColLOUGH, C. (1965). Color adaptation of edge-detectors in the human visual system. Science, 149, 1115-1116.

MichaEL, C. R. (1978a). Color vision mechanisms in monkey striate cortex: Dual-opponent cells with concentric receptive fields. Joumal of Neurophysiology, 41, 572-588.

MichaEL, C. R. (1978b). Color-vision mechanisms in monkey striate cortex: Simple cells with dual-opponent color receptive fields. Journal of Neurophysiology, 41, 1233-1249.

Morrone, M. C., Burr, D. C., \& MAFFeI, L. (1982). Functional implications of cross-orientation inhibition of cortical visual cells: $I$. Neurophysiological evidence. Proceedings of the Royal Society of London, B 216, 335-354.

Murch, G. M., \& Hirsch, J. (1972). The McCollough effect created by complementary afterimages. American Journal of Psychology, 85 , 241-247.

OVER, R. (1977). Contingent aftereffects in the perception of colour and contour. In R. H. Day \& G. V. Stanley (Eds.), Studies in perception. Perth: University of Western Australia Press.

RICHARDS, W. (1977). Lessons in constancy from neurophysiology. In W. Epstein (Ed.), Stability and constancy in visual perception: Mechanisms and processes. New York: Wiley. 
RigGs, L. A., White, K. D., \& EIMAs, P. E. (1974). Establishment and decay of orientation-contingent aftereffects of color. Perception \& Psychophysics, 16, 535-542.

STROMEYeR, C. F., III. (1978). Form-color aftereffects in human vision. In R. Held, H. W. Leibowitz, \& H.-L. Teuber (Eds.), Handbook of Sensory Physiology: Vol. VII. Perception. Berlin: SpringerVerlag.

STromeYer, C. F., III. (1984). Orientation-specific color aftereffects and simultaneous color contrast. In L. Spillmann \& B. R. Wooten,
(Eds.), Sensory experience, adaptation, and perception. Hillsdale, NJ: Erlbaum.

Thorell, L. C., De Valors, R. L., \& Albrecht, D. G. (1984). Spatial mapping of monkey V1 cells with pure color and luminance stimuli. Vision Research, 24, 751-769.

(Manuscript received May 23, 1986;

revision accepted for publication December 17, 1986.) 